\title{
Prediction of Resillience Based on Self-Compassion and Empathy in Veterans
}

\section{ART ICLE INF O}

\section{Article Type}

Descriptive Study

\section{Authors}

Mahmoodi H.* PhD
*Psychology Department, Humanities Faculty, Golestan University, Gorgan, Iran

\section{*Correspondence}

Address: Faculty of Humanities \& Social Sciences, Golestan University, Shahid Beheshti Street, Gorgan, Iran. Postal Code: 1575941138

Phone: +98 (19) 81840726

Fax: -

mahmoudi.hiva@gmail.com

\section{Article History}

Received: February 24, 2018

Accepted: August 07, 2018

ePublished: October 11, 2018

\section{A B S T R A C T}

Aims War is one of the influential factors in the public health of the community, which causes a lot of physical and mental harms in people. The aim of this study was to predict the resilience based on self-compassion and empathy in veterans of 8 years of imposed war.

Instruments \& Methods This descriptive-correlational study was conducted in 2017 among all veterans with physical and mental injuries caused by the war in Saqqez. Using proportionate stratified random sampling, 297 people (262 men and 35 women) were selected as sample. For data collection, Connor-Davidson Resilience Scale, Davis's Empathy Questionnaire, and Neff's Self-Compassion Questionnaire were used. The data were analyzed by Pearson correlation, multiple regression analysis, using SPSS 22 software.

Findings There was a positive and significant correlation among resilience with empathy $(r=0.69 ; p<0.01)$, self-compassion $(r=0.78$; $p<0.01)$, be kind to yourself $(r=0.81 ; p<0.01)$, selfconfidence $(r=0.73 ; p<0.01)$, humanity's commonality $(r=0.69 ; p<0.01)$, isolation $(r=0.50$; $\mathrm{p}<0.01)$, mindfulness $(\mathrm{r}=0.61 ; \mathrm{p}<0.01)$, and over-identifying $(\mathrm{r}=0.87 ; \mathrm{p}<0.01)$. Also, there was a positive and significant correlation between resilience and empathic concern $(r=0.61$; $\mathrm{p}<0.01)$ and perspectivism $(\mathrm{r}=0.51 ; \mathrm{p}<0.01)$ and a reverse and significant correlation between resilience and personal disturbance $(\mathrm{r}=-0.78 ; \mathrm{p}<0.01)$. Self-compassion and empathy were able to predict $71 \%$ and $60 \%$ of the variance of resilience changes in veterans, respectively, and among these two variables, self-compassion was more effective than empathy.

Conclusion The veterans with high self-compassion and empathy are more resilient.strategy in veterans with PTSD.

Keywords Resilience; Self-Compassion; Empathy; Veterans

\section{I T A T I O N L I N K S}

[1] Mental health in a population of veterans and their ... [2] Development of a new resilience scale: The Conner ... [3] Strengthening family ... [4] Building resilience during life stages ... [5] Preliminary study of resilience based group therapy for improving the ... [6] Defense and safety: Their function in ... [7] A pilot study and randomized controlled ... [8] Enhancing resilience in children ... [9] Self-compassion and adaptive psychological ... [10] Modeling of victim resilience and their family based on cognitive and ... [11] Selfcompassion in the face of shame and body image ... [12] Self-compassion and adaptive psychological ... [13] Self-compassion as a prospective predictor of PTSD symptom ... [14] Psychobiological mechanisms of resilience and ... [15] Empathy: Its ultimate and proximate ... [16] Mindfulness and counseling self-efficacy: The ... [17] Changes in dispositional empathy in American ... [18] Empathy and self-efficacy, and resiliency: An exploratory study of counseling students in ... [19] Self-compassion in patients with persistent musculoskeletal pain ... [20] Exploration of resilience construct in PTSD severity and ... [21] Psychological resilience in OEF-OIF ... [22] Relationship between attachment styles and ... [23] Self-compassion as a moderator of thinness-related ... [24] Resilience and vulnerability to adverse ... [25] Expression level of depression and psychological stress in caring for ... [26] Factors affecting resilience in subjects ... [27] Empathic concern and the muscular ... [28] Investigating the role of empathy and maxiolistic beliefs in the adequacy and social inability of 9-12-year-old ... [29] Relationship between personality type $\mathrm{D}$, self-compassion and social support with health behaviors ... [30] Assessing strengths, resilience, and growth to guide ... [31] The psychology ... [32] Mindfulness: Theoretical ... [33] Effectiveness of group empathy ... [34] Training health professionals in caring for dying ... [35] Investigating the effect of role ... [36] Resilience over the lifespan: Developmental perspectives ... 


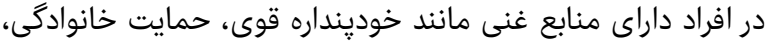

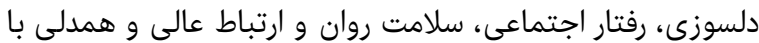

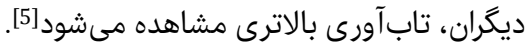

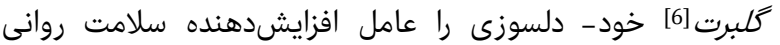

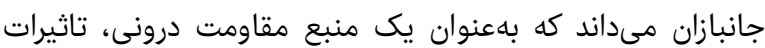

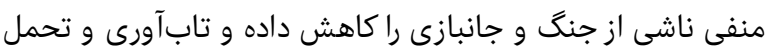

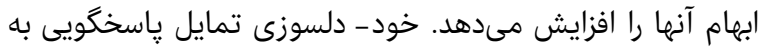

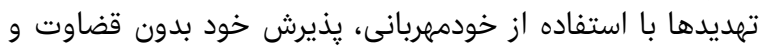

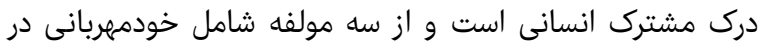

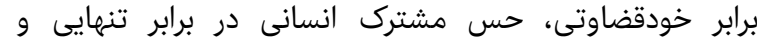

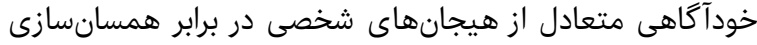

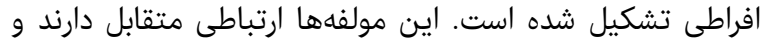

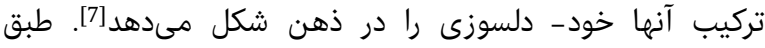

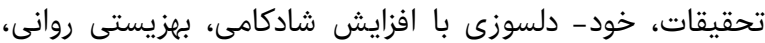

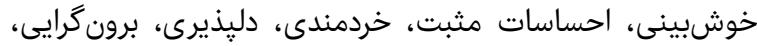

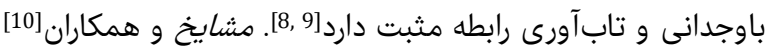

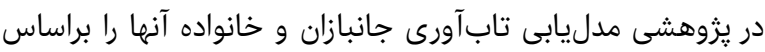

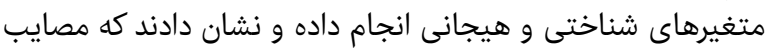

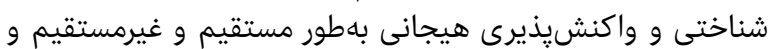

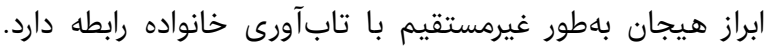

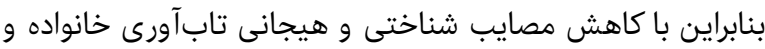

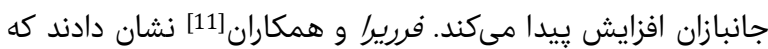

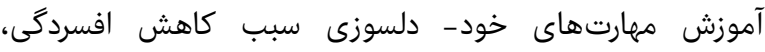

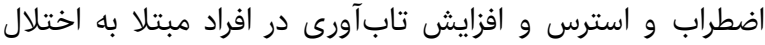

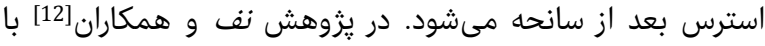

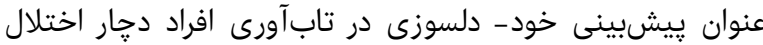

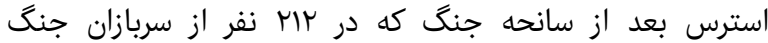

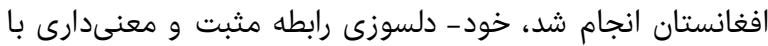

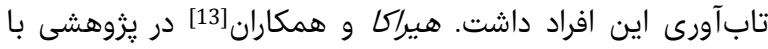

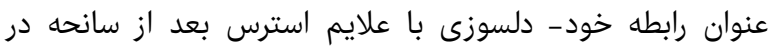

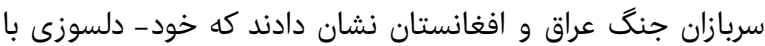

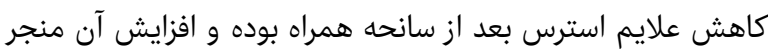

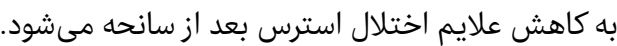

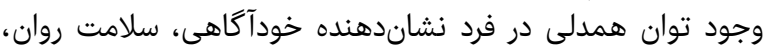
ارزش قايلشدن براى خود و دوست دوداشتن خود خدود (نه بهمعنى

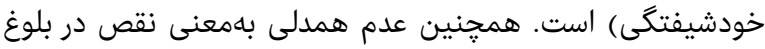

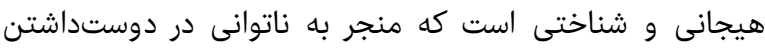

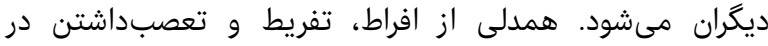

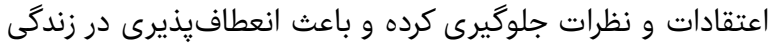

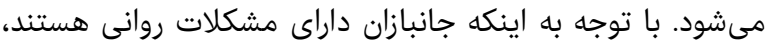

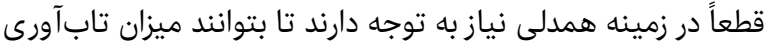

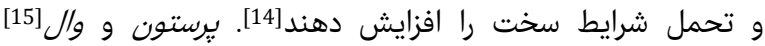

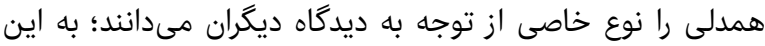

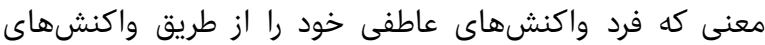

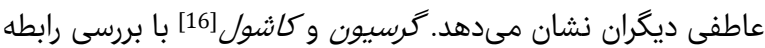

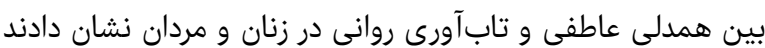

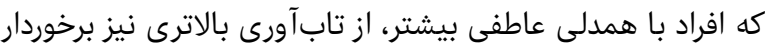

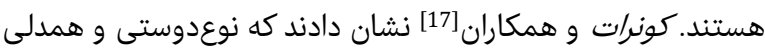

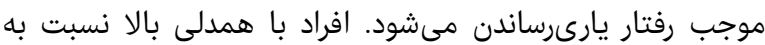

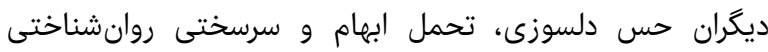

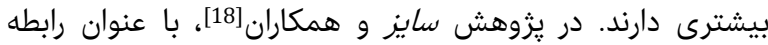

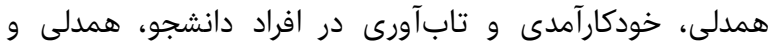

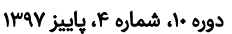

\section{ييشبينى تابآورى براساس خود- دلسوزى و

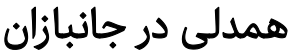

PhD " هيوا محمودى كروه روانشناسى، دانشكده علوم انسانى، دانشكاه گَلستان، گَرَان، ايران

جكيده

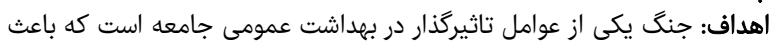

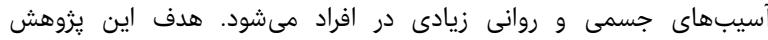

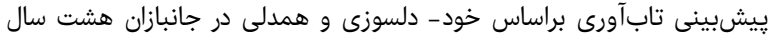

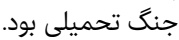

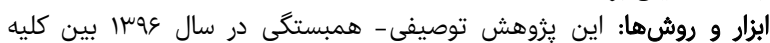

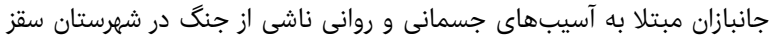

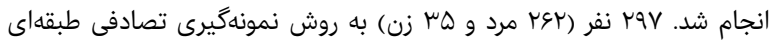

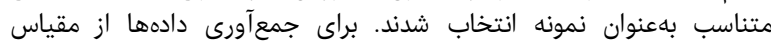

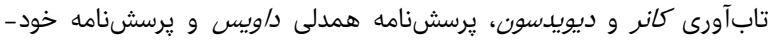

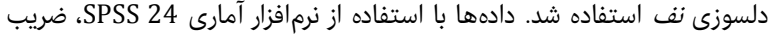

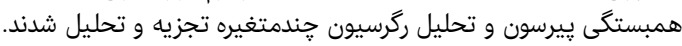

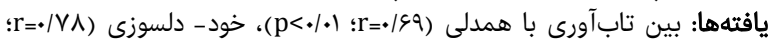

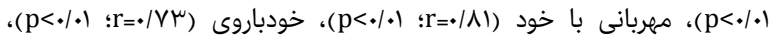

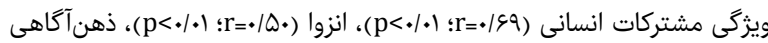

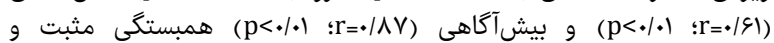

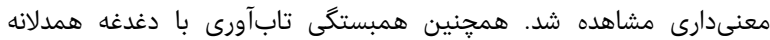

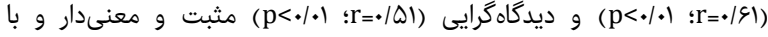

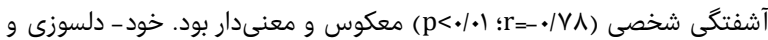

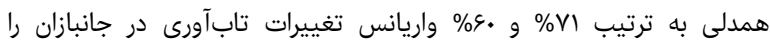

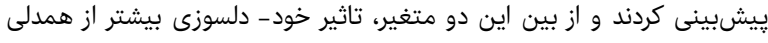
نتيجهكيرى: جانبازان داراى خود- دلسوزى و همدلى بالا، تابآورى بيشترى

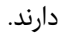
كليدواثهها: تابآورى، خود- دلسوزى، همدلى، جانبازان

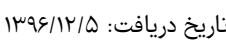

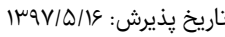

mahmoudi.hiva@gmail.com :نويسنده مسئول: تاريخ:

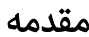

جنگ يكى از عوامل مهم و تاثيرگذار در بهداشت عمومى جامعه إنهي

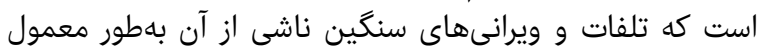

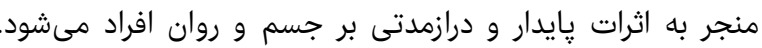

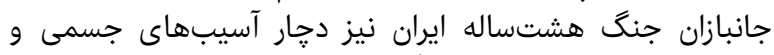

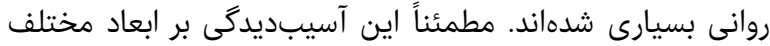

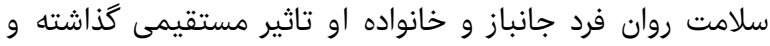

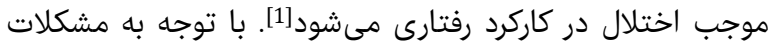

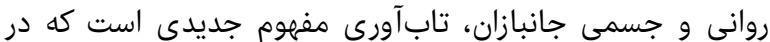

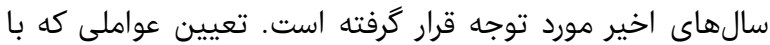

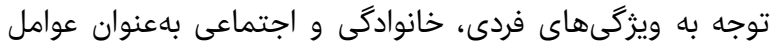

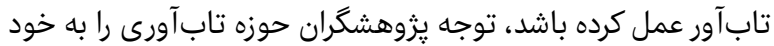

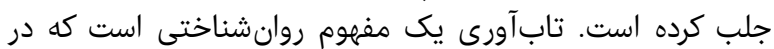

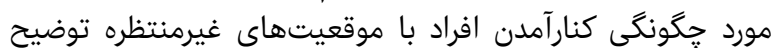

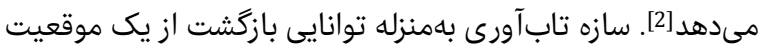

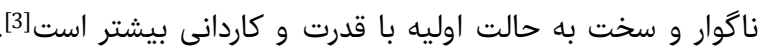

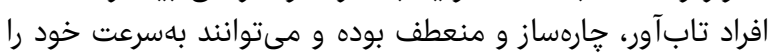

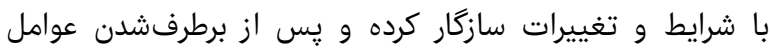

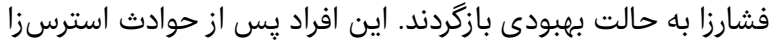

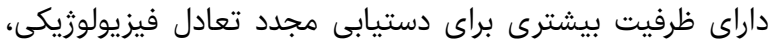

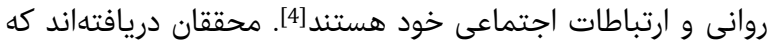




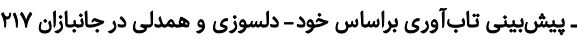

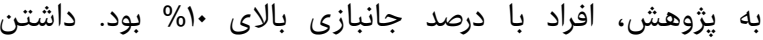

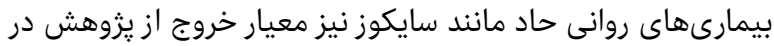

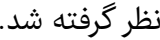

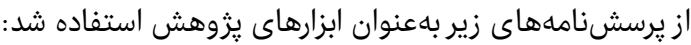
1) مقياس تابآورى كانر و ديويدسون: اين مقياس براس براى سنجش إنش

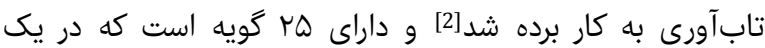

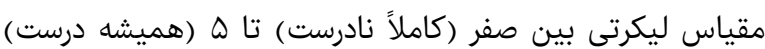

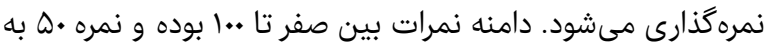

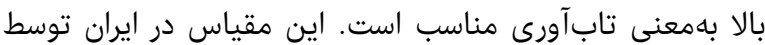

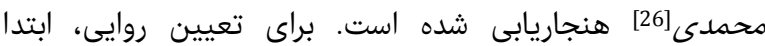

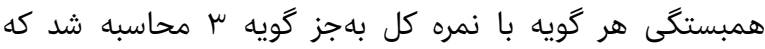

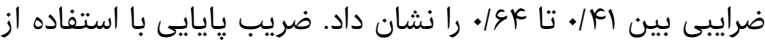

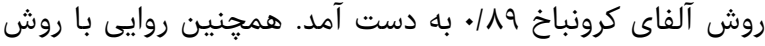

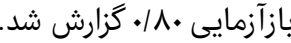

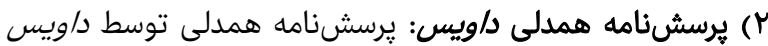

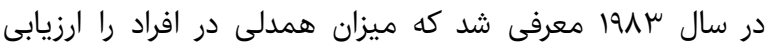

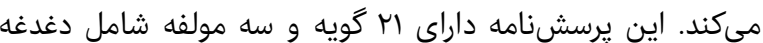

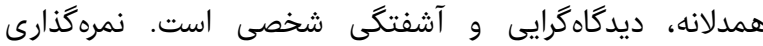

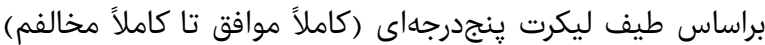

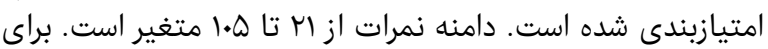

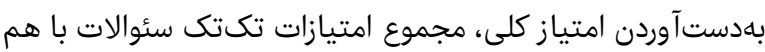

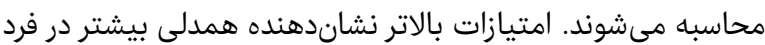

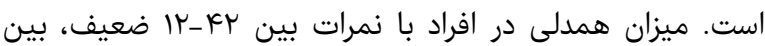

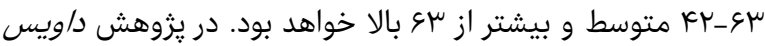

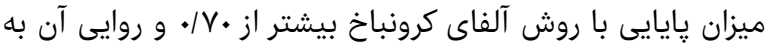

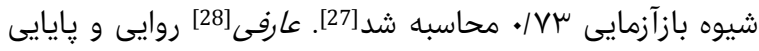

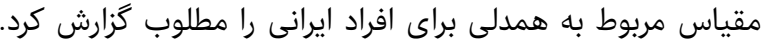

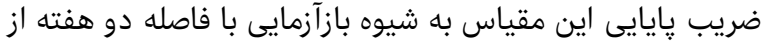

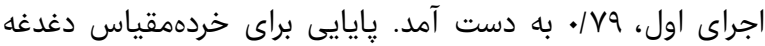

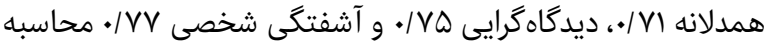
شد. همبستگى منفى ميان نمرات شاخص همدلى با يرخاشكرى و همبستخى مثبت با رفتار جامعلهيسند (r=-•/Kr)

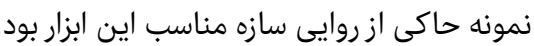

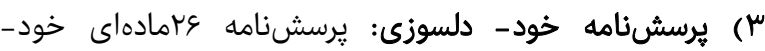

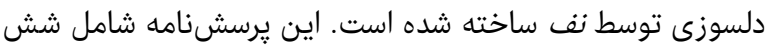

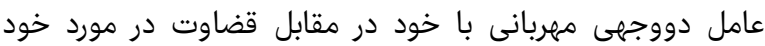

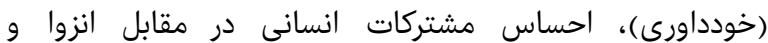

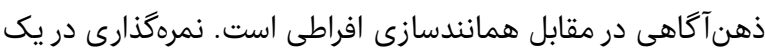

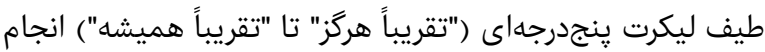

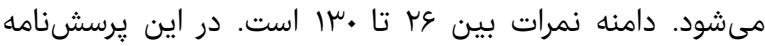

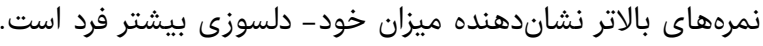

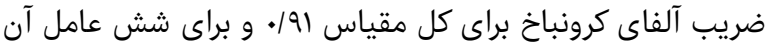

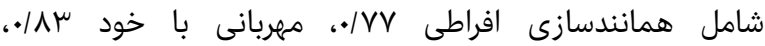

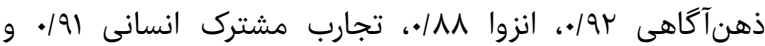

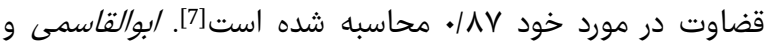

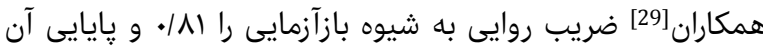

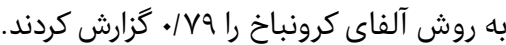

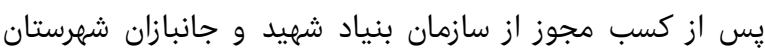

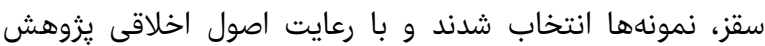

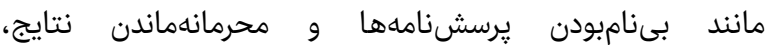

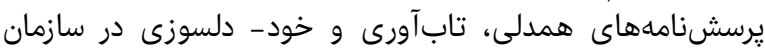
بنياد شهيد و جانبازان بهصورت فمهي، فردى توزيع و طى سو سه هفته
خودكارآمدى با تابآورى رابطه مثبت و معنىدارى داشت. ورن و

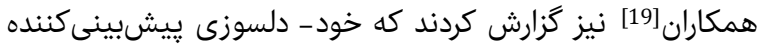

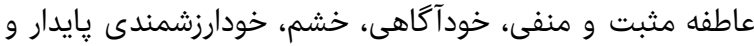

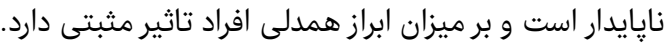

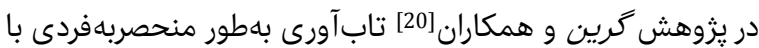

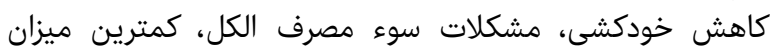

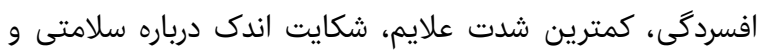

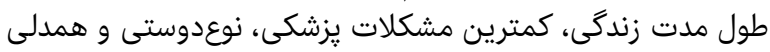

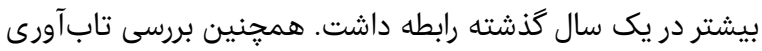

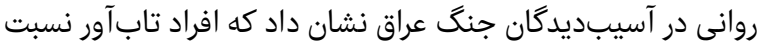

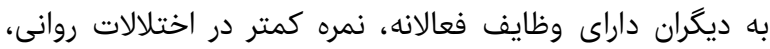

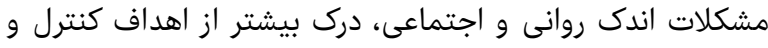

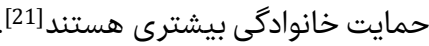

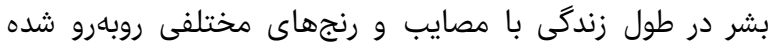

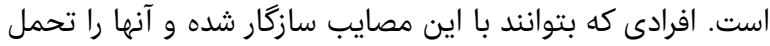

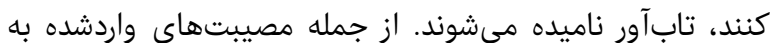

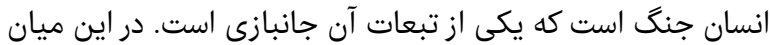

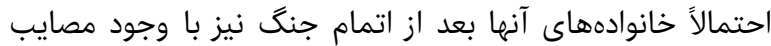

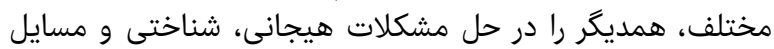

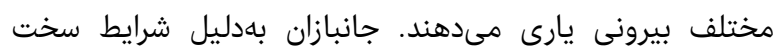

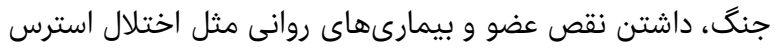

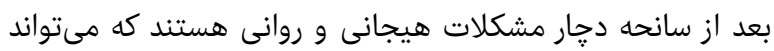

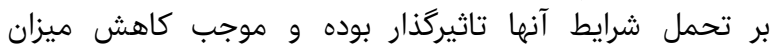

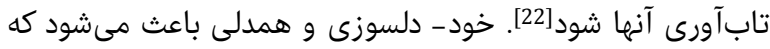

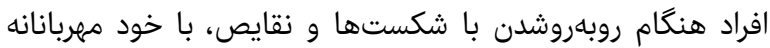

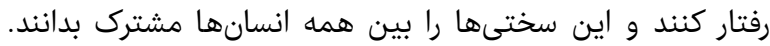

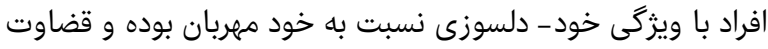

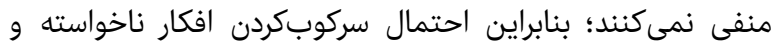

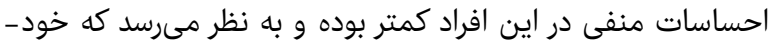

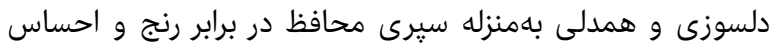

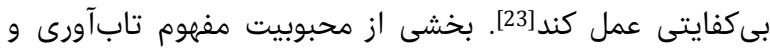

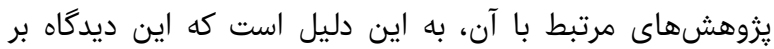

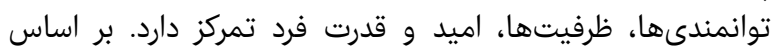

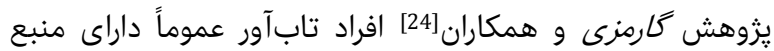

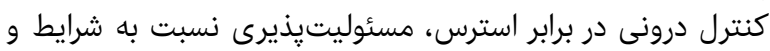

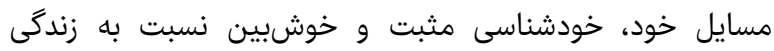

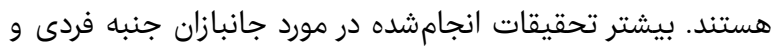

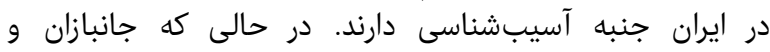

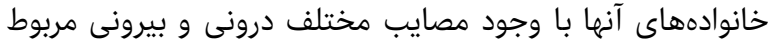

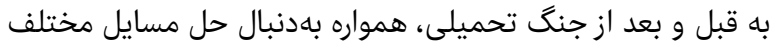

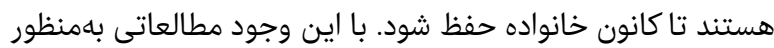

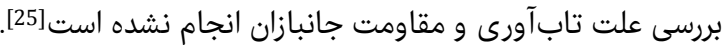

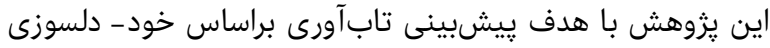

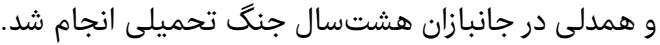

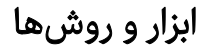
اين يزوهش توصيفى از نوع همبستخى است كه در سال عهسا در

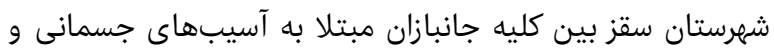

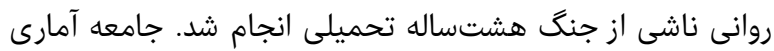

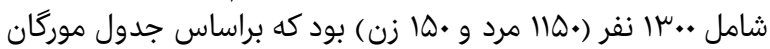

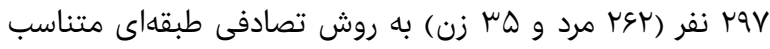

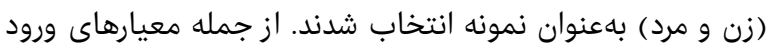


ضريب بتاى سرع/.ـ تاثير منفى بيشترى روى تابآورى جانبازان

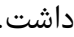

بحث

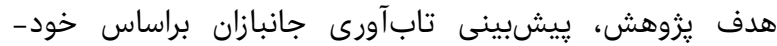

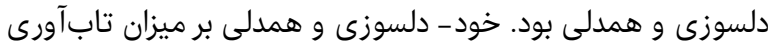

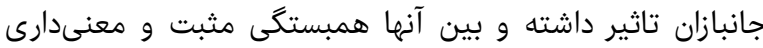

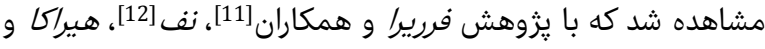

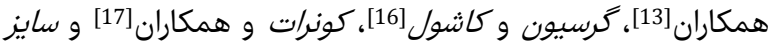

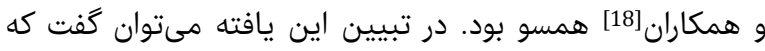

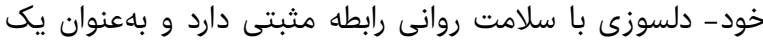

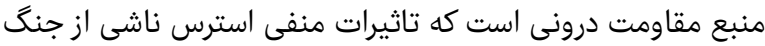

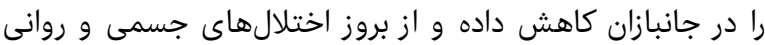

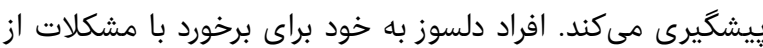

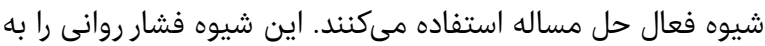

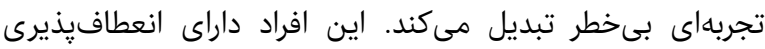

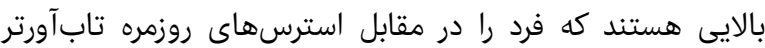

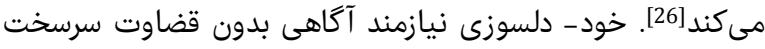

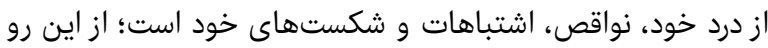

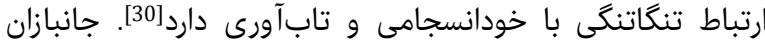

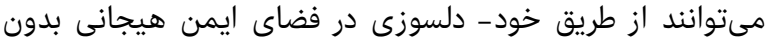

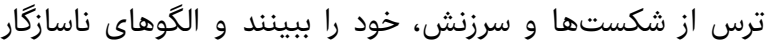

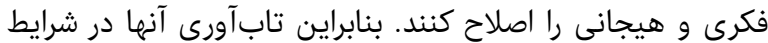

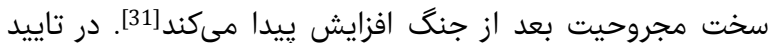

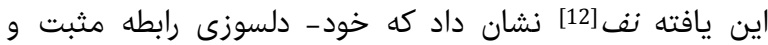

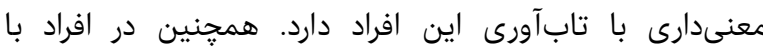

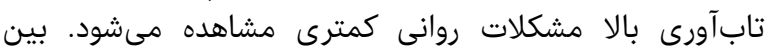

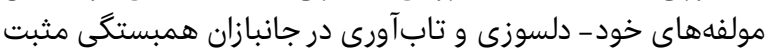

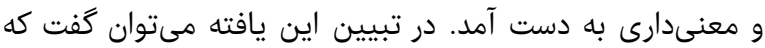

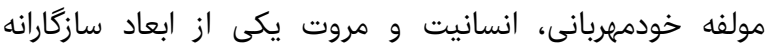

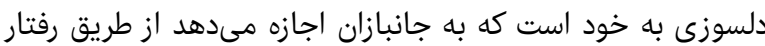

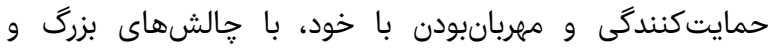

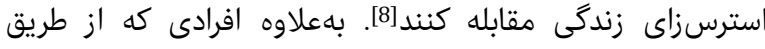

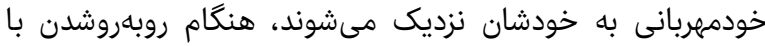

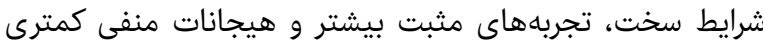

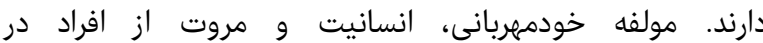

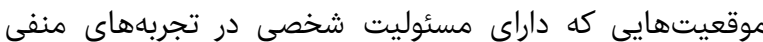

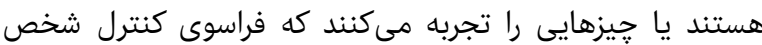

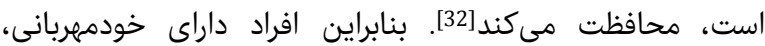

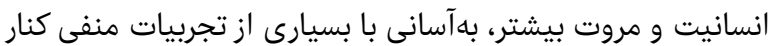

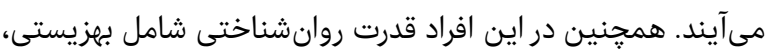

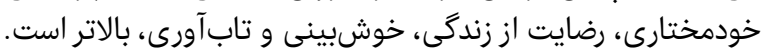

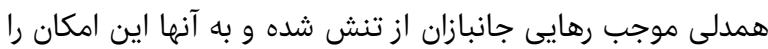

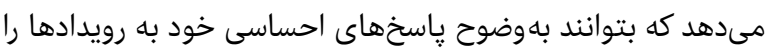

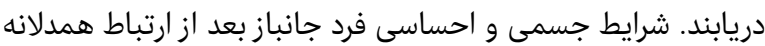

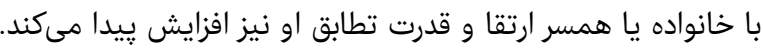

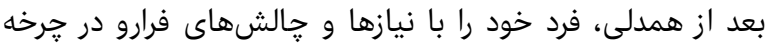

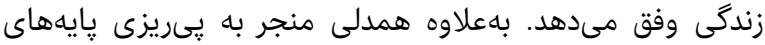

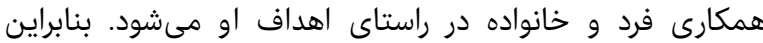

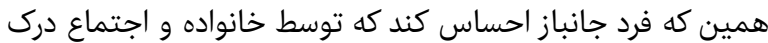

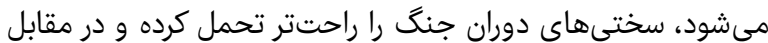

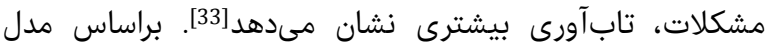
دوره •.ا، شماره F، هاييز Vqسו

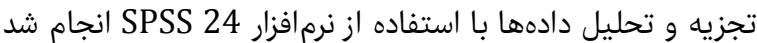

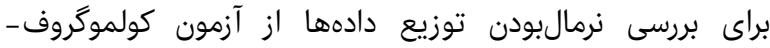

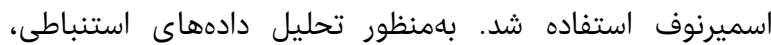

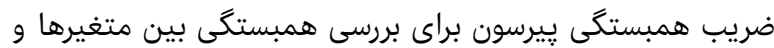

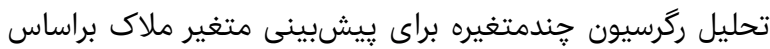

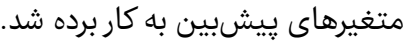

يافتهها

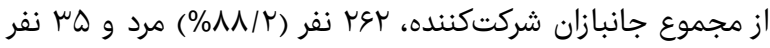

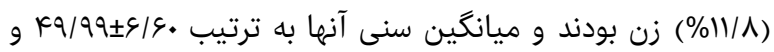

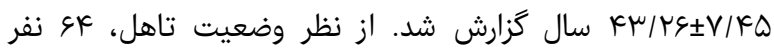

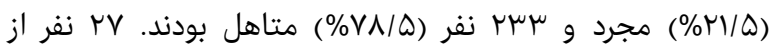

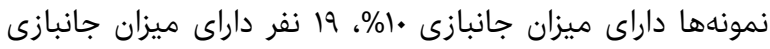

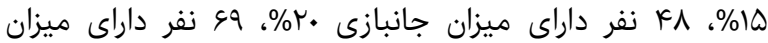

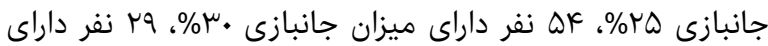

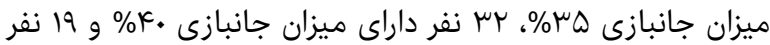

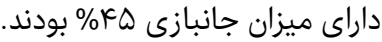

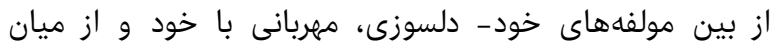

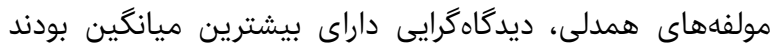

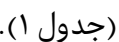

جدول () ميانگين آمارى نمرات متغيرهاى تابآورى، خود- دلسوزى، همدلى و

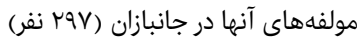

\begin{tabular}{|c|c|}
\hline نمرات & متغيرها \\
\hline & تابآورى \\
\hline \multirow[t]{2}{*}{$7 \mu / 01 \pm 11 / \cdot \Lambda$} & كل \\
\hline & خود- دلسوزى \\
\hline$V / 7 V \pm 7 / 0$. & مهربانى با خود \\
\hline $0 / .7 \pm r / \varepsilon r$ & خودداورى \\
\hline$T / \cdot \Lambda \pm r / \varepsilon O$ & ويزگى مشترك انسانى \\
\hline $0 / 7 \varepsilon \pm \mu / 07$ & انزوا \\
\hline $0 / 0 V \pm r / V$. & ذهن آكاهى \\
\hline$\mu / \varepsilon r \pm 1 / \tau \Lambda$ & بيشآكاهى \\
\hline \multirow[t]{2}{*}{$70 / \mu q \pm q / v$. } & كل \\
\hline & همدلى \\
\hline$r \mu / O \Lambda \pm \mu / V O$ & دغدغه همدلانه \\
\hline$r\urcorner / \mu \varepsilon \pm \mu / \Lambda q$ & ديدگًامرايى \\
\hline $\mid r / \| r \pm r / A V$ & آشفتخى شخصى \\
\hline$T V / 0 \varepsilon \pm 1 / \cdot \varepsilon$ & كل \\
\hline
\end{tabular}

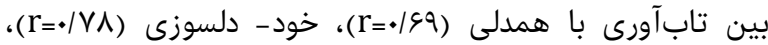

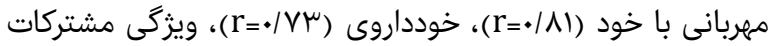

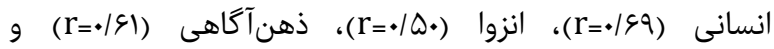

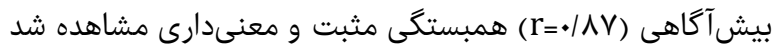

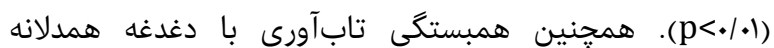

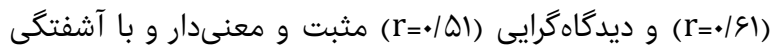
(r=-•/VA)

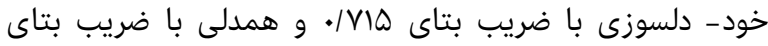

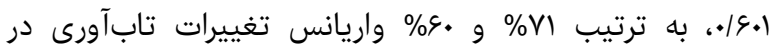

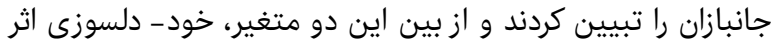

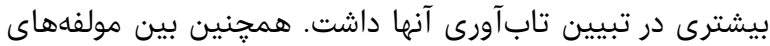

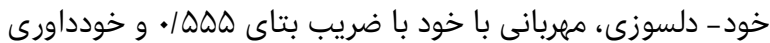

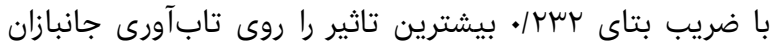

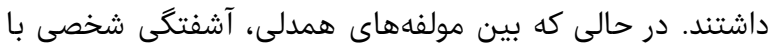




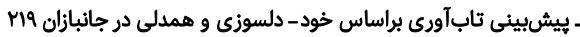

3- Walsh F. Strengthening family resilience. 3rd Edition. New York and London: Guilford Press; 2016. pp. 23-6.

4- Anasuri S. Building resilience during life stages: Status and strategies. Int J Humanit Soc Sci. 2016;6(3):1-9.

5- Watson CC, Rich BA, Sanchez L, O’Brien K, Alvord MK. Preliminary study of resilience based group therapy for improving the functioning of anxious children. J Child Youth Care Forum. 2014;43(3):269-86.

6- Gilbert P. Defense and safety: Their function in social behavior and psychopathology. $\mathrm{Br} \mathrm{J}$ Clin Psychol. 1993;32(Pt2):131-53.

7- Neff KD, Germer CK. A pilot study and randomized controlled trial of the Mindful Self-Compassion Program. J Clin Psychol. 2013;69(1):28-44.

8- Alvord MK, Grados JJ. Enhancing resilience in children: A proactive approach. Prof Psychol: Res Pract. 2005;36(3):238-45.

9- Neff KD, KirkpatrickKL, Rude SS. Self-compassion and adaptive psychological functioning. J Res Personal. 2007;41(1):139-54.

10- Mashayekh M, Borjali A, Delavar A, Shafi Abadi A. Modeling of victim resilience and their family based on cognitive and emotional variables. J Mil Psychol. 2012;2(8):1-23. [Persian]

11- Ferreira C, Pinto-Gouveia J, Duarte C. Selfcompassion in the face of shame and body image dissatisfaction: Implications for eating disorders. Eat Behav. 2013;14(2):207-10.

12- Neff KD, Kirkpatrick KL, Rude SS. Self-compassion and adaptive psychological functioning. J Res Personal. 2007;41(1):139-54.

13- Hiraoka R, Meyer EC, Kimbrel NA, DeBeer BB, Gulliver SB, Morissette SB. Self-compassion as a prospective predictor of PTSD symptom severity among trauma-exposed US Iraq and Afghanistan war veterans. J Trauma Stress. 2015;28(2):127-33.

14- Charney DS. Psychobiological mechanisms of resilience and vulnerability: Implications for successful adaption to extreme stress. AM J Psychiatry. 2004;161(2):195-216.

15- Preston SD, De Waal FB. Empathy: Its ultimate and proximate bases. Behav Brain Sci. 2002;25(1):1-71.

16- Greason PB, Cashwell CS. Mindfulness and counseling self-efficacy: The mediating role of attention and empathy. Couns Educ Superv. 2009;49(1):2-19.

17- Konrath SH, O’Brien EH, Hsing C. Changes in dispositional empathy in American college students over time: A meta-analysis. Pers Soc Psychol Rev. 2011;15(2):180-98.

18- Siyez DM, Savi F. Empathy and self-efficacy, and resiliency: An exploratory study of counseling students in Turkey. Proced Soc Behav Sci. 2010;5(2):459-63.

19- Wren AA, Somers TJ, Wright MA, Goetz MC, Leary MR, Fras AM, et al. Self-compassion in patients with persistent musculoskeletal pain: Relationship of selfcompassion to adjustment to persistent pain. J Pain Symptom Manage. 2012;43(4):759-70.

20- Greene KT, Calhoun PS, Dennis MF, Beckham JC. Exploration of resilience construct in PTSD severity and functional correlates in military combat veteran who have served since September 11, 2001. J Clin psychiatry. 2010;71(7):823-30.

21- Pietrzak RH, Southwick SM. Psychological resilience in OEF-OIF Veterans: Application of a novel classification approach and examination of demographic and psychosocial correlates. J Affect Disord.
فشنباخ، افرادى كه همدلى بالايى دارند، در تشخيص نشانههاى

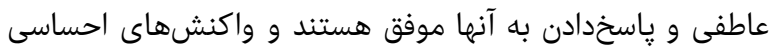

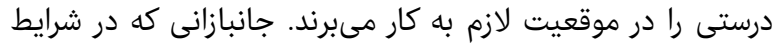

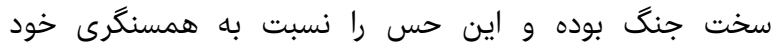

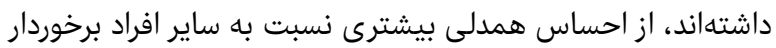

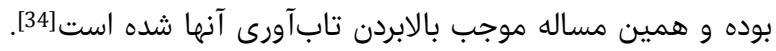

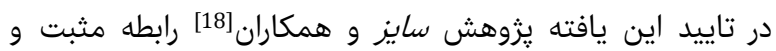

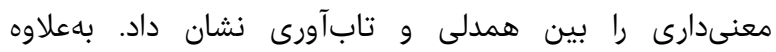

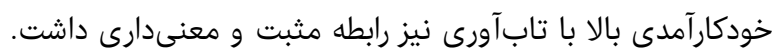

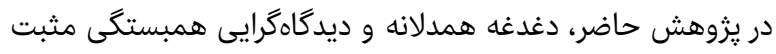

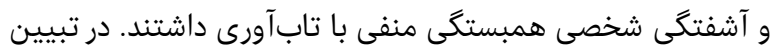

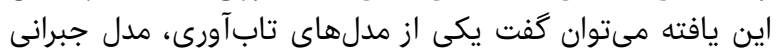

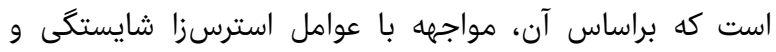

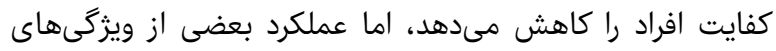

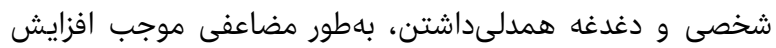

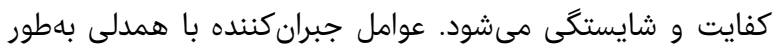

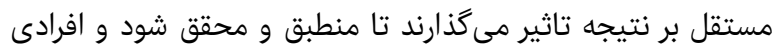

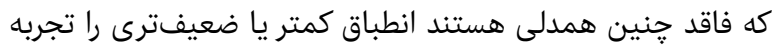

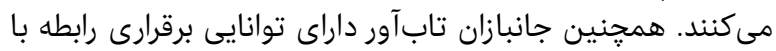

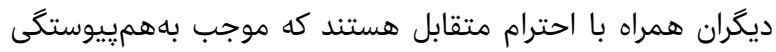

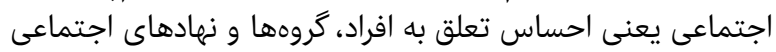

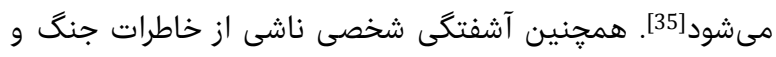

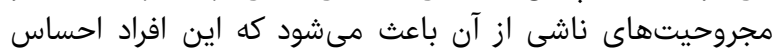

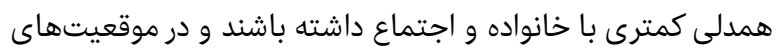
مختلف نيز تاب آورى كمترى داشته بانه باشند.

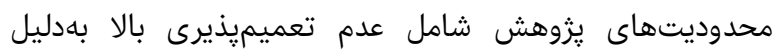

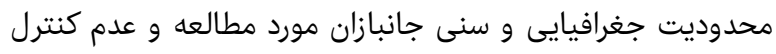

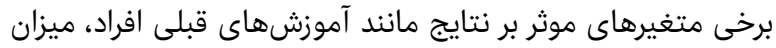

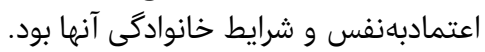

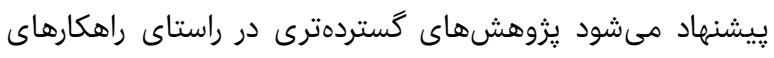
افزايش ميزان همدلى در خانوادههاى جانبازان انجام شود ئرد

نتيجهگيرى

جانبازان داراى خود- دلسوزى و همدلى بالا، تابآورى بيشترى دارند.

تشكر و قدردانى: از همكارى جانبازان عزيز و حمايتهاى مسئولان

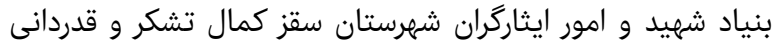

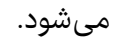
تاييديه اخلاقى: قبل از اجراى يزوهش، هماهنكگىهاى لازم با بنياد

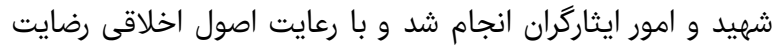

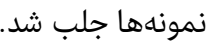
تعارض منافع: موردى از سوى نويسنده ذئ ذكر نشده است.

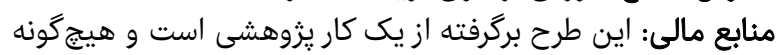

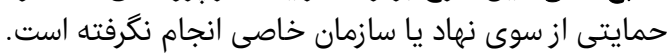

منابع

1- Bahreinian SA, Borhani H. Mental health in a population of veterans and their wives in Qom province. Res Med. 2001;27(4):305-12. [Persian]

2- Conner KM, Davidson JR. Development of a new resilience scale: The Conner-Davidson Resilience scale (CD-RISC). Depress Anxiety. 2003;18(2):76-82. 
29- Abolghasemi A, Taghipour M, Narimani M. Relationship between personality type D, selfcompassion and social support with health behaviors in coronary heart disease patients. Health Psychol. 2011;1(1):5-19. [Persian]

30- Tedschi RG, Kilmer RP. Assessing strengths, resilience, and growth to guide clinical interventions. Prof Psychol: Res Pract. 2005;36(3):230-7.

31- Wispé L. The psychology of sympathy. 1st Edition. New York: Springer US; 1991.

32- Brown KW, Ryan RM, Creswell JD. Mindfulness: Theoretical foundations and evidence for its salutary effects. Psychol inq. 2007;18(4):211-37.

33- Refahi ZH, Moghtaderi N. Effectiveness of group empathy training on the increase of couples' intimacy. Fam Couns Psychother. 2012;3(1):44-55. [Persian] 34- Papadatou D. Training health professionals in caring for dying children and grieving families. Death Stud. 1997;21(6):575-600.

35- Madi Neshat M, Lashkardost H, Tabatabaeichehr M. Investigating the effect of role play scenarios on changing mood and empathy of nursing students in psychiatry. Strides Dev Med Educ. 2012;10(4):431-8. [Persian]

36- Masten AS, Wright MOD. Resilience over the lifespan: Developmental perspectives on resistance, recovery and transformation. In: Reich JW, Zautra AJ, Hall JS, editors. Handbook of adult resilience. New York: Guilford Press; 2010. pp. 213-37.
2011;133(3):560-8.

22- Akbari Z, Vafaei T, Khosravi S. Relationship between attachment styles and the resiliency amount of veterans wives. Iran J War Public Health. 2011;3(2):12-7. [Persian]

23- Tylka TL, Russell HL, Neal AA. Self-compassion as a moderator of thinness-related pressures' associations with thin ideal internalization and disordered eating. Eat Behav. 2015;17:23-6.

24- Garmezy N. Resilience and vulnerability to adverse developmental outcomes associate with poverty. Am Behav Sci. 1991;34(4):416-30.

25- Manteghi A, Hebrani P, Samari AA, Heydari AS. Expression level of depression and psychological stress in caring for spouses of veterans hospitalized in psychiatry and its relation with the rate of hospitalization. J Fundam Ment Health. 2010;12(45):410-9.

26- Mohammadi M. Factors affecting resilience in subjects at risk of substance abuse [Dissertation]. Tehran: University of Social Welfare and Rehabilitation Sciences; 2006. [Persian]

27- Davis MH. Empathic concern and the muscular dystrophy telethon: Empathy as a multidimensional construct. Personal Soc Psychol Bull. 1983;9(2):223-9.

28- Arefi M, Latifian M. Investigating the role of empathy and maxiolistic beliefs in the adequacy and social inability of 9-12-year-old students. J Educ Learn Stud. 2011;3(1):61-82. [Persian] 